Der Comet war immer sehr blass und schlecht zu beohachten; vorzüglich gilt dieses von den Dech.-Beobachtungen. Die Heliometer-Einstellungen am $4^{\text {ten }}$ Decbr. waren so wenig befriedigend, dass ich durch Benutzung der Kreise des Instruments bessere Resultate erzielt $\mathrm{zu}$ haben glaube, die nothwendig bei der Schwäche des noch $1 \frac{1}{2}-2^{\prime}$ grossen, fast gleichförmig hellen Objectes eine nicht unbeträchtliche Unsicherheit involviren. Zur Pointirung auf Stern und Comet bediente ich mich eines Netzes von Metallfäden, die ohne jegliche Erleuchtung in dunklen Felde wahrnehmbar sind

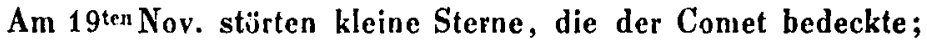
einige Minuten lang sah ich mich während der centralen Bedeckung eines Sternes $12.13^{\mathrm{m}}$ genöthigt, die Vergleichungen aufzugeben, da ich durchaus nicht im Stande war, den Stern von dem verdichteten centralen Theile des Cometennebels zu unterscheiden. Die Rectascension Decbr. 9 ist unsicher, weil wegen plötzlicher Trübung für diese Coordinate nur drei Einstellungen gelangen.

N y s a. (Fortsetzung von A.N. 1092 u. 1098).
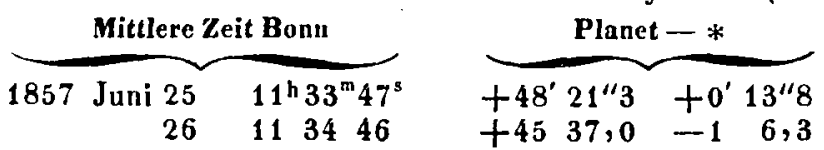

Der an beiden Tagen benutzte Vergleichstern $10^{\mathrm{m}}$ wurde mit einem benachbarten Sterne $9^{m}$, den Herr Prot. Argelander am 24 Juni folgendermaassen in Meridiane beobachtet hat:

$$
\alpha=14^{\mathrm{l}} 57^{\mathrm{m}} 10^{\mathrm{s}} 73 \quad \delta=-11^{\circ} 50^{\prime} 16^{\prime \prime} 37
$$
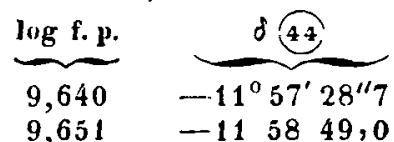

$-11^{\circ} 57^{\prime} 28^{\prime \prime} 7$

$-115849,0$

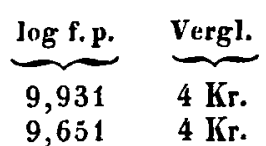

heliometrisch verbunden, wodurch sich folgende Relationen ergaben :

Juni $23 \quad$ Dist. $=530^{\prime \prime 77} \quad$ Pos.-W. $=212^{\circ} 44^{\prime} 24$

Juni $24 \quad$ Dist. $=530,32 \quad$ Pos. $-W .=21243,54$

\begin{tabular}{|c|c|c|}
\hline \multicolumn{3}{|c|}{ Mittlere Zeit Boun } \\
\hline $1857 \mathrm{Juli}$ & 14 & $11^{\mathrm{h}} 36^{\mathrm{m}} 0^{\mathrm{s}}$ \\
\hline & 18 & 11352 \\
\hline & 26 & $\begin{array}{lll}10 & 19 & 9\end{array}$ \\
\hline & 27 & 11034 \\
\hline
\end{tabular}

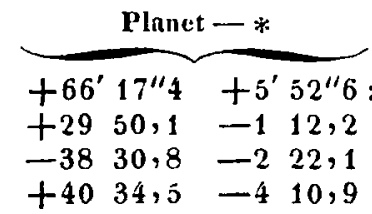

Mittlere Oerter der Vergleichsterne 1857,0:

Juli $14 \alpha=243^{\circ} 29^{\prime} 40^{\prime \prime} 1 \quad \delta=-12^{\circ} 34^{\prime} 46^{\prime \prime} 5 \quad$ B.Z. Z. 246

$\begin{array}{rrrrrrrl}18 & 244 & 1 & 55,9 & -12 & 42 & 47,0 & \text { B.Z. } 246 \\ 26 & 245 & 21 & 2,4 & -13 & 15 & 4,2 & \text { BZ., LI.29972 } \\ 27 & 254 & 5 & 16,7 & -13 & 17 & 42,4 & \text { BZ., LI.29834 }\end{array}$

lch habe vorgezogen, den Planeten nicht mit dem von Herru Goldschmidt gewählten Namen zu bezeichnen, da dieser schwerlich allgenein angenommen werden dürfte.

Die Positionen der Vergleichsterne zu den obigen Beobachtungen sind auf den Argelander'schen Catalog bezogen. Ein h hinter der Zahl der Vergleichungen bedeutet, dass das Heliometer als solches gebraucht wurde; Kr. bezeichnet Kreismicrometerbeobachtungen, zu denen zurveilen die Schwäiche der Objecte nöthigte.
Planet 45 .

\begin{tabular}{|c|c|c|c|c|}
\hline$\alpha \longdiv { 4 5 }$ & $\log \mathbf{f} . p$ & $8 \overparen{45}$ & $\log \mathbf{f} . \mathbf{p}$ & Vergl. \\
\hline $244^{\circ} 36^{\prime} 38^{\prime \prime} 6$ & 9,639 & $-12^{0} 29^{\prime} 6^{\prime \prime} 5:$ & 9,930 & $3 \mathbf{K r}$. \\
\hline 2443226,6 & 9,602 & $\begin{array}{llll}-12 & 44 & 11,3\end{array}$ & 9,937 & \\
\hline 2444312,0 & 9,568 & $\begin{array}{llll}-13 & 17 & 37,2\end{array}$ & 9,941 & 8 \\
\hline $244 \quad 46 \quad 39,9$ & 9,670 & -1322 & 9,932 & 8 \\
\hline
\end{tabular}

Die ersten Beobachtungen des Cometen 1. 1857, die hier schärfer reducirt nochmals aufgreführt sind, hat Hr. Dr. Schulze mit seinen Elementen verglichen; er hat dabei den A.N. 1077 angezeigten Druckfehler in der Zeit der ersten Beobachtung vom $2^{\text {ten }}$ März ühersehen. Verbessert man ihn, so geben die beiden Positionen einen sehr nahe gleichen Fehler der Elemente. -

Die Beohachtungen des Cometen Il. 1857 werde ich veräffentlichen, sobald Herr Professor Argelander die Vergleichsterne wird bestimmen können, wozu er sich freundlichst crhoten; ich habe im Ganzen 16 Positionen erhalten, wovon 8 in die Zeit Mai 13-23 fallen. Es wird von Interesse sein zu schen, inwieweit diese Heliometerbeohachtungen mit den übrigen Bestimmungen harmoniren.

\section{A. Winnecke.}

\title{
Schreiben des Herrn J.Ferguson an den Herausgeber.
}

On examining the reduction of some Comet-observations sent to you by Mr. Maury on the $18^{\text {th }}$ I found that I had mistaken one of the stars of comparison, and that some of the reductions had been erroneously computed.

I therefore hasten to send you the enclosed corrected observations. -

$I$ have not been able to see the Comet since the $17^{\text {tb }}$.

Washington 1857 Nov. 23.

J. Ferguson. 


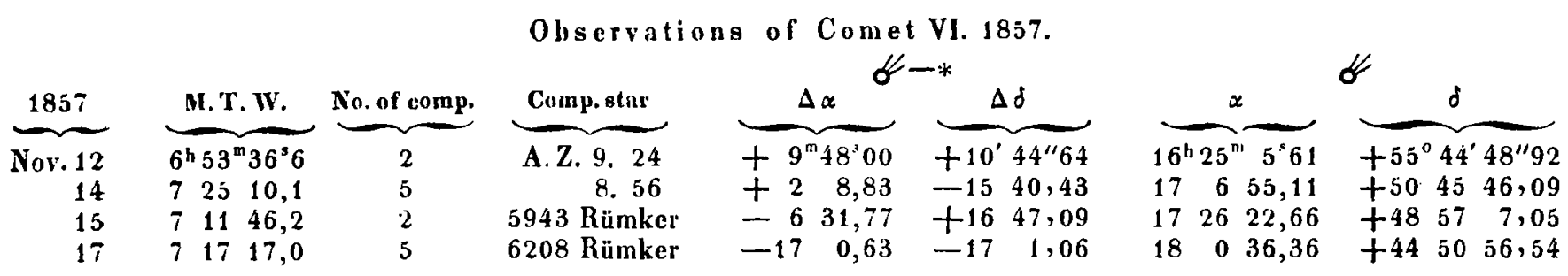

Adopted mean places for 1860,0 of Comparison stars:

\begin{tabular}{|c|c|c|c|c|c|c|}
\hline A. \%. 9.24 & 7 & $16^{h} 12$ & $22^{s} 53$ & $+53^{\circ} 33$ & $47^{\prime \prime} 09$ & \multirow{2}{*}{ Argelander's Zones } \\
\hline 8. 56 & 7 & 174 & 51,32 & 511 & $16,55\}$ & \\
\hline 5943 Rünker & 6 & 1732 & 58,21 & $48 \quad 40$ & $6,09\}$ & \multirow{2}{*}{ Rümker's Catalogue } \\
\hline 6208 Rümker & 8 & 1758 & 39,71 & $45 \quad 40$ & $6,09\}$ & \\
\hline
\end{tabular}

Beobachtungen des Cometen IV.1857, von Hern Dr. Förster.

\begin{tabular}{|c|c|c|c|c|c|c|c|c|}
\hline 1857 & M. Zt. Berlin & & $\alpha$ & app. & $\log f \cdot p$ & c & app. & $\log f . p$ \\
\hline Juli 30 & $12^{h} 14^{\mathrm{m}} 19^{\mathrm{y}}$ & 66 & 54 & $2 " 9$ & $0,9131 u$ & +54 & $35^{\prime} 39^{\prime \prime} 6$ & 0,7524 \\
\hline Aug. 1 & $\begin{array}{lll}11 & 29 & 22\end{array}$ & 71 & 39 & 34,2 & $0,8237 n$ & +52 & 1847,6 & 0,8267 \\
\hline 3 & 112940 & 76 & 3 & 21,2 & $0,7853 n$ & +49 & $49 \quad 7,9$ & 0,8448 \\
\hline 3 & $14 \quad 1434$ & 76 & 17 & 29,6 & $0,9077 n$ & +49 & 4036,6 & 0,6410 \\
\hline 4 & $\begin{array}{lll}12 & 11 & 13\end{array}$ & 78 & 9 & 21,2 & $0,8259 n$ & +48 & 2859,1 & 0,8161 \\
\hline 8 & $12 \quad 2258$ & 85 & 26 & 0,8 & $0,7817 n$ & +43 & 218,9 & 0,8350 \\
\hline 11 & 125230 & 90 & 8 & 39,0 & $0,7781 n$ & +38 & 1,3 & 0,8286 \\
\hline 12 & 134430 & 91 & 38 & 6,7 & $0,8049 n$ & +37 & 2156,0 & 0,7884 \\
\hline 13 & $13 \quad 1436$ & 92 & 59 & 26,6 & $0,7877 n$ & +35 & 5947,0 & 0,8208 \\
\hline 15 & $14 \quad 1124$ & 95 & 40 & 33,5 & $0,7909 n$ & +33 & 1029,6 & 0,7843 \\
\hline 20 & $14 \quad 18 \quad 21$ & 101 & 36 & 3,3 & $0,7629 u$ & +26 & 3026,0 & 0,8041 \\
\hline 23 & $\begin{array}{lll}14 & 28 & 27\end{array}$ & 104 & 48 & 47,6 & $0,7558 n$ & +22 & 4444,2 & 0,8085 \\
\hline 29 & 15120 & 110 & 40 & 47,7 & $0,7339 u$ & +15 & 538,9 & 0,8090 \\
\hline Sept. 2 & $\begin{array}{lll}15 & 31 & 12\end{array}$ & 114 & 16 & 14,8 & $0,7169 n$ & +11 & 4912,7 & 0,8056 \\
\hline 7 & $\begin{array}{lll}15 & 46 & 1\end{array}$ & 118 & 26 & 12,6 & $0,7062 u$ & +7 & 6,5 & 0,8156 \\
\hline
\end{tabular}

Berlin 1857 Dec. 12.

W. Förster.

Aus einem Schreiben des Herrn Dr. C. H. F. Peters an den Herausgeber.

Vielleicht dürften die folgenden beiden Beobachtungen des von Van Arsdale am $11^{\text {ten }}$ d.M. entdeckten Cometen Ihnen von Werth sein, besonders für den Fall, dass der Comet nicht auch unabhängig in Europa aufgefunden sein sollte:*)

\begin{tabular}{|c|c|c|c|}
\hline 1857 & Albany Stemzeit & $\mathbf{A R} \not \dot{~}$ & Decl. of \\
\hline & $\begin{array}{l}22^{h} 33^{n 1} 12^{h} \\
223543\end{array}$ & 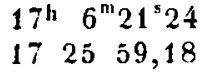 & $\begin{array}{r}+50^{\circ} 49^{\prime} 1^{\prime \prime} 5 \\
485915,3\end{array}$ \\
\hline
\end{tabular}

Die Beobachtungen sind am Ringnikrometer des Cometensuchers gemacht, und bei der Reduction die eigne Bewegung des Cometen noch nicht berücksichtigt. Vergleichstern war an ersten Abend Piazai XVII. 19 (im Mittel aus Piazzi, Argelander und Radcliffe Observations), am zweiten B.A.C.597j

*) Der Comet ist bekanntlich von Herru Dr. Donati cinige Stunden früher entdeckt, als von Herru Van Arsdale. u. Piazzi XVII. 166, welcher letztere Stern auch von Lalande beobachtet worden, aber in Baily's Catalog 1 Minute in Decl. unrichtig angegeben ist. - Die absolute Zeit dürfte vielleicht noch eine Correction erfahren, vielleicht bia zu 10 Secunden, da die Küstenvermessung ihr kleines Transit zurückgenommen und ich zur Zeitbestimmung aul einen kleinen Sextanten beschränkt hin.

Für den am $25^{\text {sten }}$ Juli von mir zuerst aufgefundenen Cometen hat Herr Watson, der talentvolle Schüler Dr. Brïnnom's, vor einiger Zeit elliptische Elemente in Astr. Journal bekannt gemacht, von denen die folgenden, welche auf Beobachtungen vom Dudley Observ. und einer vom $23^{\text {sten }}$ Sept., welche ich in Cambridge gemeinschaftlich mit Herrn George $\boldsymbol{P}$. Bond am grossen Refractor anzustellen das Vergnügen batte, beruben, nicht sehr abweichen: 